\title{
Ideal-lens stars
}

Jakub Bĕlín, Tomáš Tyc, Stephen Oxburgh, Johannes Courtial

Jakub Bĕlín, Tomáš Tyc, Stephen Oxburgh, Johannes Courtial, "Ideal-lens stars," Proc. SPIE 10376, Novel Optical Systems Design and Optimization XX, 1037605 (24 August 2017); doi: 10.1117/12.2272991

SPIE Event: SPIE Optical Engineering + Applications, 2017, San Diego, California, SPIE. United States 


\title{
Ideal-lens stars
}

\author{
Jakub Bělín ${ }^{\mathrm{a}}$, Tomáš Tyc ${ }^{\mathrm{b}}$, Stephen Oxburgh ${ }^{\mathrm{a}}$, and Johannes Courtial ${ }^{\mathrm{a}}$

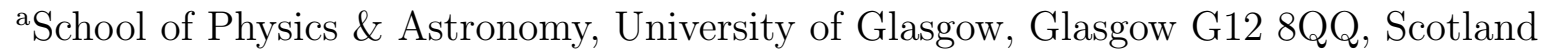 \\ b Institute of Theoretical Physics and Astrophysics, Masaryk University, Kotlarska 2, 61137 \\ Brno, Czech Republic
}

\begin{abstract}
We recently showed how structures of ideal (thin) lenses can act as (ray-optical) transformation-optics devices. This was done by breaking the structure down into all sets of ideal lenses in the structure that share a common edge, and showing that these sets have very specific imaging properties.

In order to start the development of a general understanding of the imaging properties of sets of ideal lenses that share a common edge, we investigate here particularly simple and symmetric examples of combinations of ideal lenses that share a common edge. We call these combinations ideal-lens stars. An ideal-lens star is formed by $N$ identical ideal lenses, each placed such that they share a principal point (which lies on the common edge) and such that the angles between all neighbouring lenses are the same.

We find that that passage through every single ideal lens in the ideal-lens star images any point to itself. Furthermore, light-ray trajectories in ideal-lens stars are piecewise linear approximations to conic sections. (In the limit of $N$ approaching infinity, they are conic sections.)
\end{abstract}

Keywords: imaging; optical design; lens systems

\section{INTRODUCTION}

We recently started to investigate structures of ideal thin lenses that form transformation-optics devices. Transformation-optics devices ${ }^{1,2}$ are defined by a coordinate transformation between physical (real) space and virtual space (or electromagnetic space), and their function is to make any object at a physical-space position appear at the corresponding virtual-space position, irrespective of the direction from which an observer positioned outside the device views the object.

The most high-profile examples of transformation-optics device are invisibility cloaks. In the simplest case, their associated coordinate transformation magnifies a virtual-space point inside the device into a finite-sized sphere in physical space. Any object placed inside this sphere will then appear to be point-sized - and therefore infinitely small - when seen from outside the cloak, and thus be hidden from view. Furthermore, any objects seen through the cloak appear at their original position, and so (neglecting absorption and other such effects) the cloak is invisible.

The idea to realise transformation-optics devices with ideal thin lenses originated from our work on pixellated, light-ray-direction-changing, sheets called telescope windows. ${ }^{3,4}$ Such sheets perform a pixellated version of generalised refraction, which has associated shortcomings such as field-of-view limitations, ${ }^{5,6}$ but on the other hand it can be shown that this enables refraction that cannot be achieved without such compromises. ${ }^{7,8}$ This very general refraction enables telescope windows to perform the most general imaging that maps between all of object space and all of image space, ${ }^{9,10}$ but it is important to realise that, due to the shortcomings of pixellation, the imaging is not stigmatic (i.e. ray-optically perfect) but merely integral (i.e. it is not individual light rays that intersect at conjugate positions but the centres of bundles of light rays).

The very general imaging properties of telescope windows enabled the construction - so far only theoretical - of transformation-optics devices consisting entirely of idealised telescope windows. The idealisation is that

Further author information: (Send correspondence to J.C.)

J.C.: E-mail: johannes.courtial@glasgow.ac.uk, Telephone: +44 (0)141 3306429 


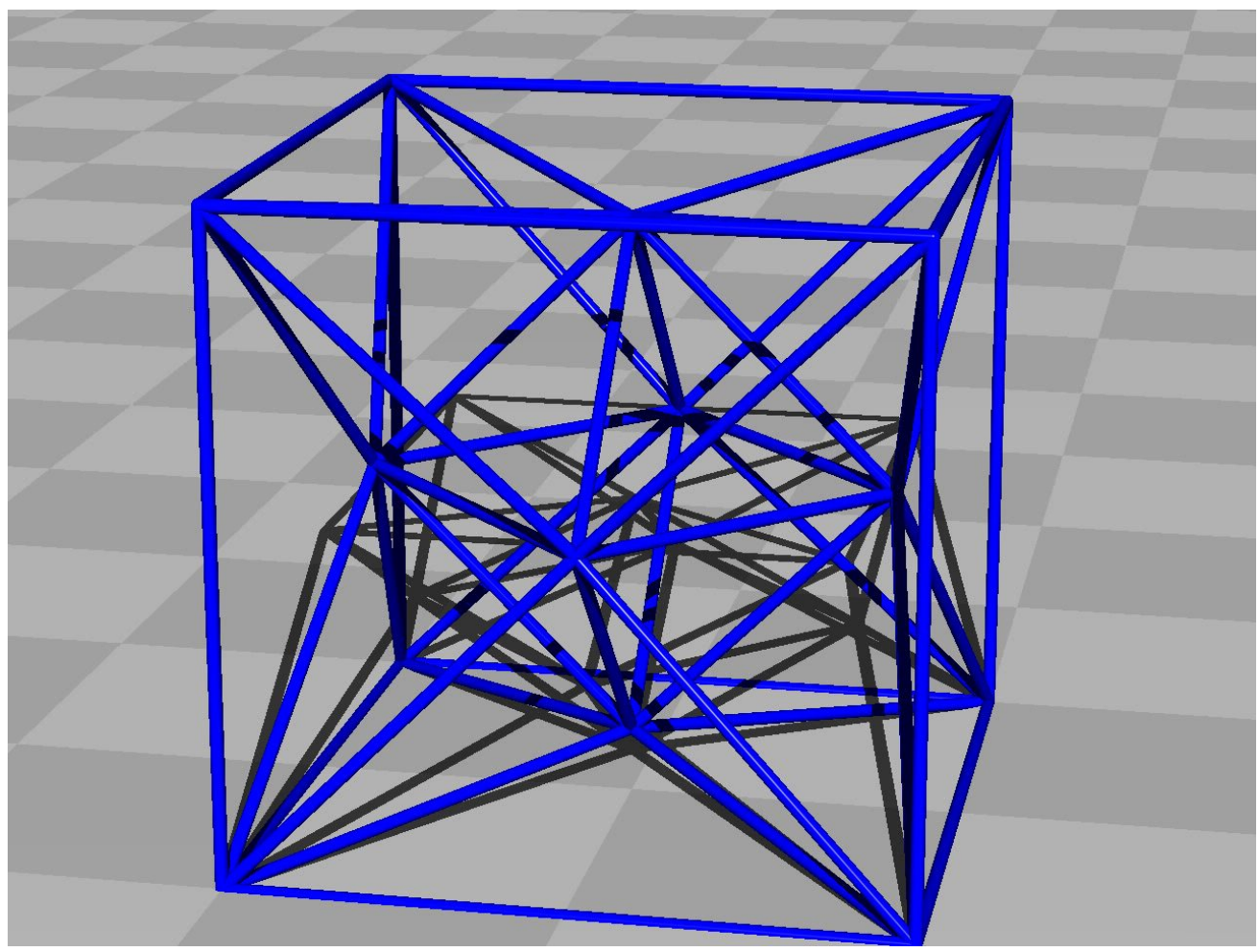

Figure 1. Structure of the. ${ }^{11}$ Each blue cylinder marks the edge of a homogeneous telescope window. There are six square telescope windows that form the faces of the outside cube, and numerous triangular telescope windows on the inside. With the right choice of telescope-window parameters, the central octahedron appears smaller when viewed from outside the cloak. The image was calculated with an extended version of the customised raytracer Dr TIM. ${ }^{12}$

all detrimental effects of pixellation are being neglected. We first investigated the simplest case, in which all telescope windows were homogeneous (meaning that every part of the telescope window changes the direction of transmitted light rays identically). ${ }^{11,14}$ Fig. 1 shows the structure of a homogeneous-telescope-window cloak.

Next, we investigated the more complicated case of devices consisting of inhomogeneous telescope windows. It transpires that the idealised inhomogeneous telescope windows that image between all of object and image space are simply ideal thin lenses generalised to have two different focal lengths on their two sides. We call such generalised thin lenses glenses. ${ }^{15}$ Fig. 2 shows the structure of a glens cloak. ${ }^{13}$

We noticed that several of the glenses in the glens cloak shown in Fig. 2 have the same focal lengths on their two sides, and so they are actually simply ideal thin lenses. We started to wonder if it is possible to construct transformation-optics devices entirely from ideal thin lenses. Such devices would elegantly avoid the shortcomings of pixellation, and they would be an omni-directional generalisation of the paraxial "Rochester cloak". ${ }^{16}$ The construction of such devices is indeed possible if certain design rules are followed; Fig. 3 shows the structure of an example, an ideal-lens cloak.

The key design rule is as follows. We place a closed loop around any one of the edges in an ideal-lens-based transformation-optics device. This loop intersects the ideal lenses intersecting at the edge. We now take an arbitrary point $\mathrm{P}$ and calculate its image $\mathrm{P}^{\prime}$ due to the lens intersected first. Next, we calculate the position of the image $\mathrm{P}^{\prime \prime}$ of $\mathrm{P}^{\prime}$ due to the lens intersected next, and continue this re-imaging process until all lenses intersected by the loop have been taken into account. The requirement then is that the eventual image position must be the same as the original position $\mathrm{P}$, for any choice of P. In other words, the combination of all lenses that meet at a common edge to image every point back to itself.

This design rule led us to investigate combinations of lenses that satisfy it, as these combinations would then be the building blocks of ideal-lens transformation-optics devices. One type of these ideal-lens combinations 


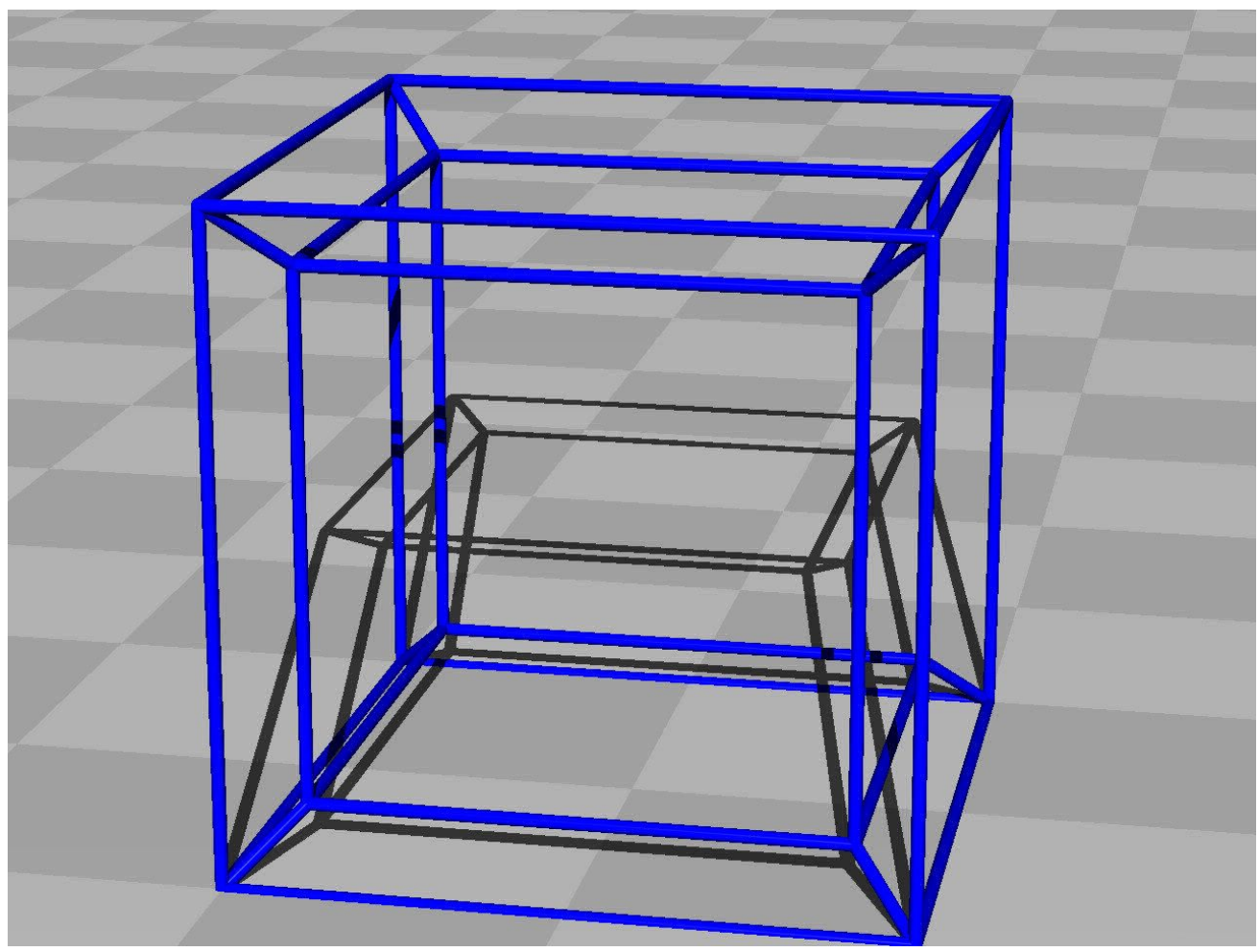

Figure 2. Structure of the glens cloak. ${ }^{13}$ The blue cylinders now mark the edges of glenses, that is, idealised inhomogeneous telescope windows. Each quadrilateral formed by four cylinders marks the area of a suitable glens. Specifically, all squares formed by cylinders (the faces of the inner and outer cubes) mark the area of glenses, the quadrilaterals connecting corresponding edges of the inner and outer cubes mark the area of ideal thin lenses. The image was calculated with the raytracer Dr TIM. ${ }^{12}$

stood out for its simplicity and symmetry. We call this type of ideal-lens combination an ideal-lens star, and investigate it here.

\section{IDEAL-LENS STARS}

Consider $N$ squares arranges such that one side of each square touches a given axis and the angles between all neighbouring squares are the same (namely $360^{\circ} / N$ ). Replacing the squares with ideal thin lenses, all with the same focal length $f$ and all sharing the same principal point (or lens centre), which lies on the axis, results in an ideal-lens star. Figs 4 and 5 show raytracing images of an ideal-lens star comprising $N=7$ ideal thin lenses.

Fig. 6 shows a number of trajectories, all originating from the same point light source, in an ideal-lens star. It can be seen that all trajectories shown intersect again at a position opposite that of the point light source, and a number of trajectories continue to intersect again at the position of the point light source, forming closed trajectories. Different trajectories are shown in different colours: the closed trajectories are blue and dark purple, the open trajectories light purple and red.

It can be shown mathematically that the trajectories in an ideal-lens star in the limit $N \rightarrow \infty$ are conic sections. Fig. 7 illustrates this, showing trajectories that start identically to those shown in Fig. 6 in an ideal-lens star with a large number of lenses. It can be seen that the closed trajectories are now very close approximations to a circle (blue trajectory) and an ellipse (dark purple), the open trajectories are now approximating a parabola (light purple) and a hyperbola (red).

Fig. 8 shows the view from a point on a closed trajectory inside the ideal-lens star shown in Fig. 4, looking in the direction of the trajectory. The trajectory is chosen to pass through the red sphere also shown in Fig. 4, 


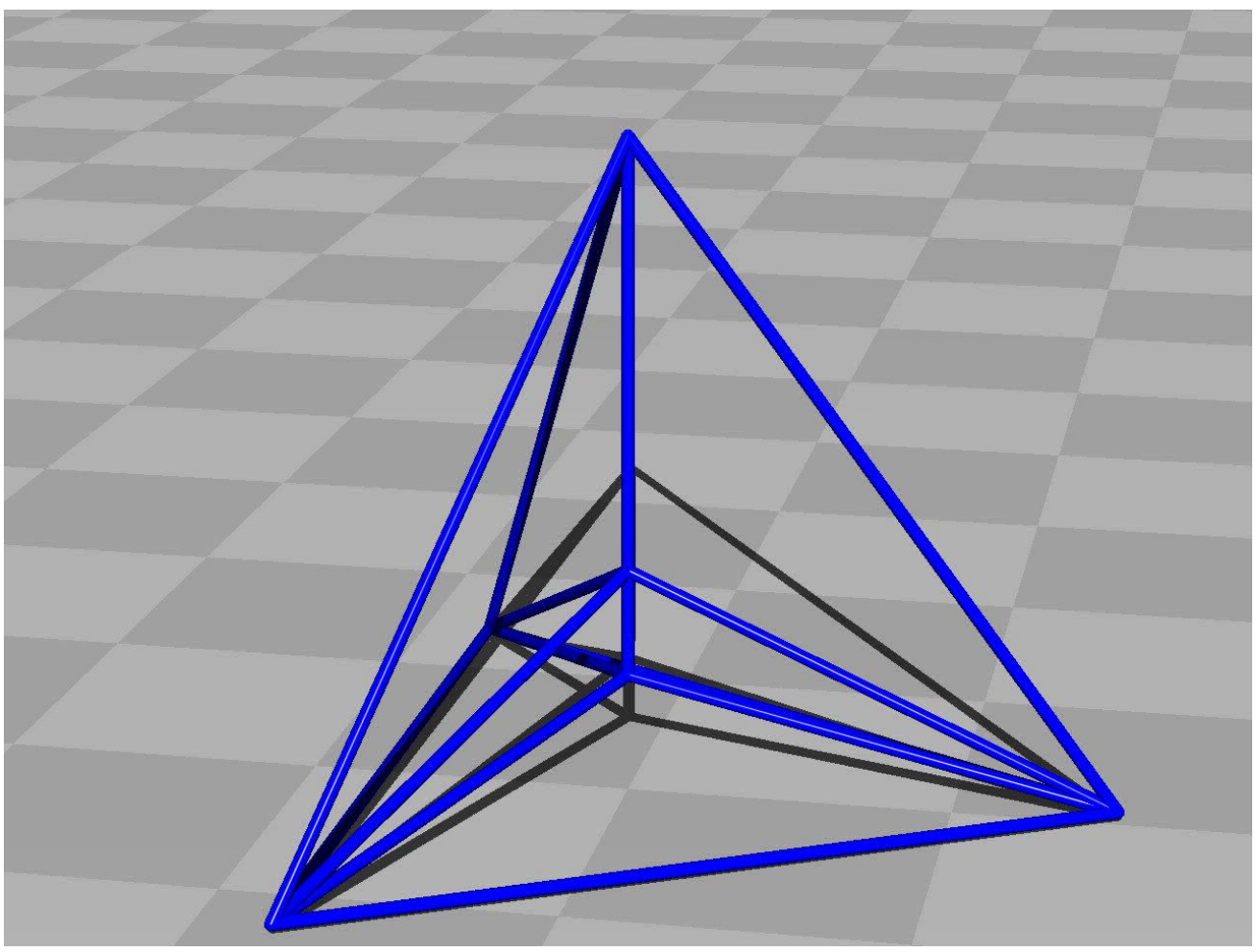

Figure 3. Structure of an ideal-lens cloak. The blue cylinders this time mark the edges of ideal thin lenses. All lenses are triangular in shape. The image was calculated with the raytracer Dr TIM. ${ }^{12}$

which is why the red sphere is visible in the centre of the image. More importantly, however, it can be seen that the view surrounding the image of the sphere is black. This is due to the fact that all light rays that reach the camera from those directions travel on closed trajectories, and as such never reach a light source, which is why the view in those directions is dark.

\section{CONCLUSIONS}

We have described a structure of ideal thin lenses called ideal-lens stars with the property that the lenses, in combination, image every point to itself. We have not derived nor proved this property here, but instead we have shown several raytracing simulations that are consistent with the predicted imaging properties. Ideal-lens stars are useful building blocks for ideal-lens-based transformation-optics devices.

An obvious question for future research is how the properties of ideal-lens stars change if the ideal thin lenses are replaced with physical lenses. These properties could then be tested experimentally.

\section{ACKNOWLEDGMENTS}

This work was supported by the UK's Engineering and Physical Sciences Research Council [grant numbers $\mathrm{EP} / \mathrm{K} 503058 / 1$ and EP/M010724/1].

\section{REFERENCES}

[1] U. Leonhardt, "Optical conformal mapping," Science 312, pp. 1777-1780, 2006.

[2] J. B. Pendry, D. Schurig, and D. R. Smith, "Controlling electromagnetic fields," Science 312, pp. 1780-1782, 2006.

[3] J. Courtial, "Ray-optical refraction with confocal lenslet arrays," New J. Phys. 10, p. 083033, 2008. 


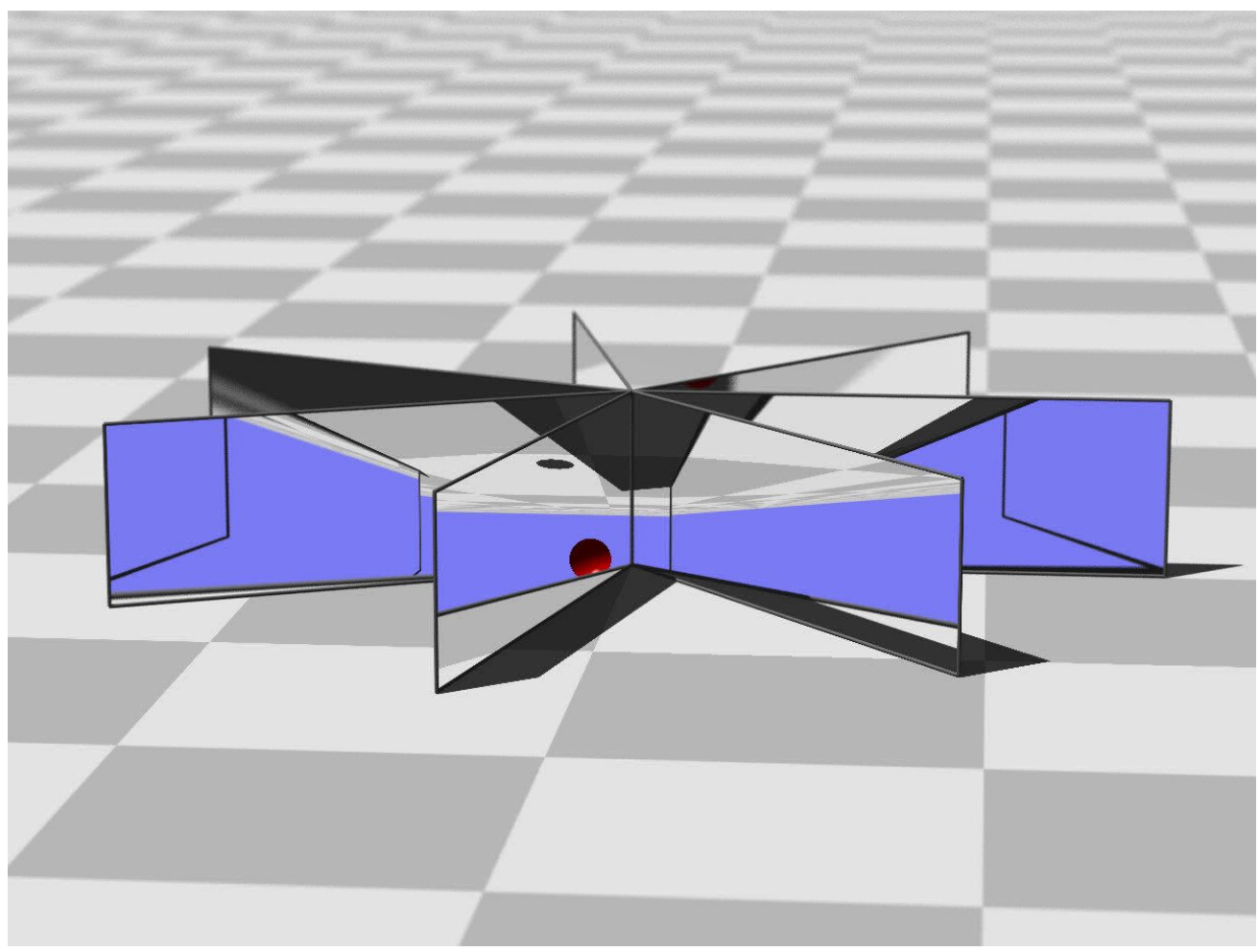

Figure 4. Lens star with $N=7$ lenses. A red sphere placed inside the star is also shown. The image was created with Dr TIM. $^{12}$ The focal length of each lens is one floor-tile width.

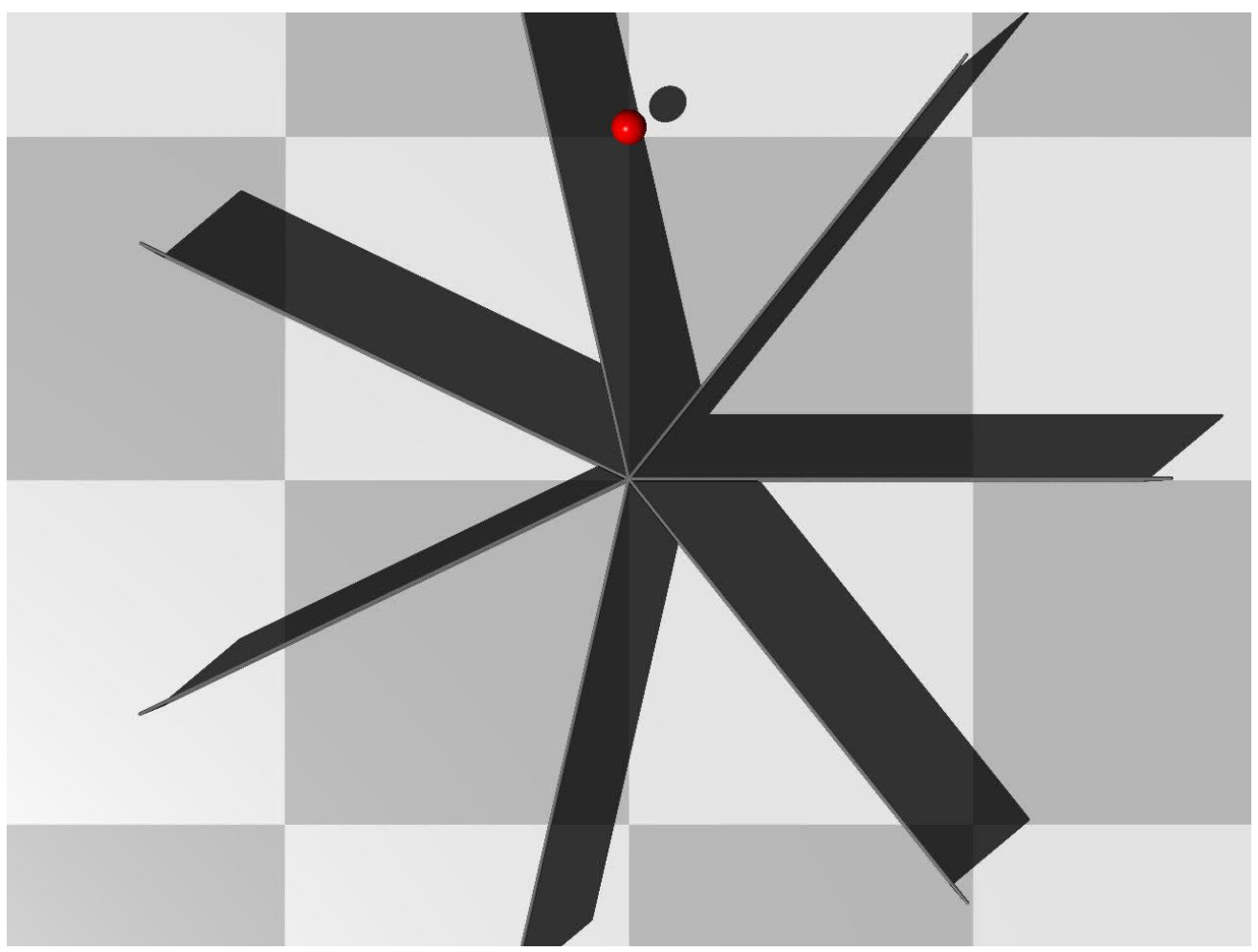

Figure 5. Top view of the lens star shown in Fig. 4. The image was created with Dr TIM. ${ }^{12}$ 


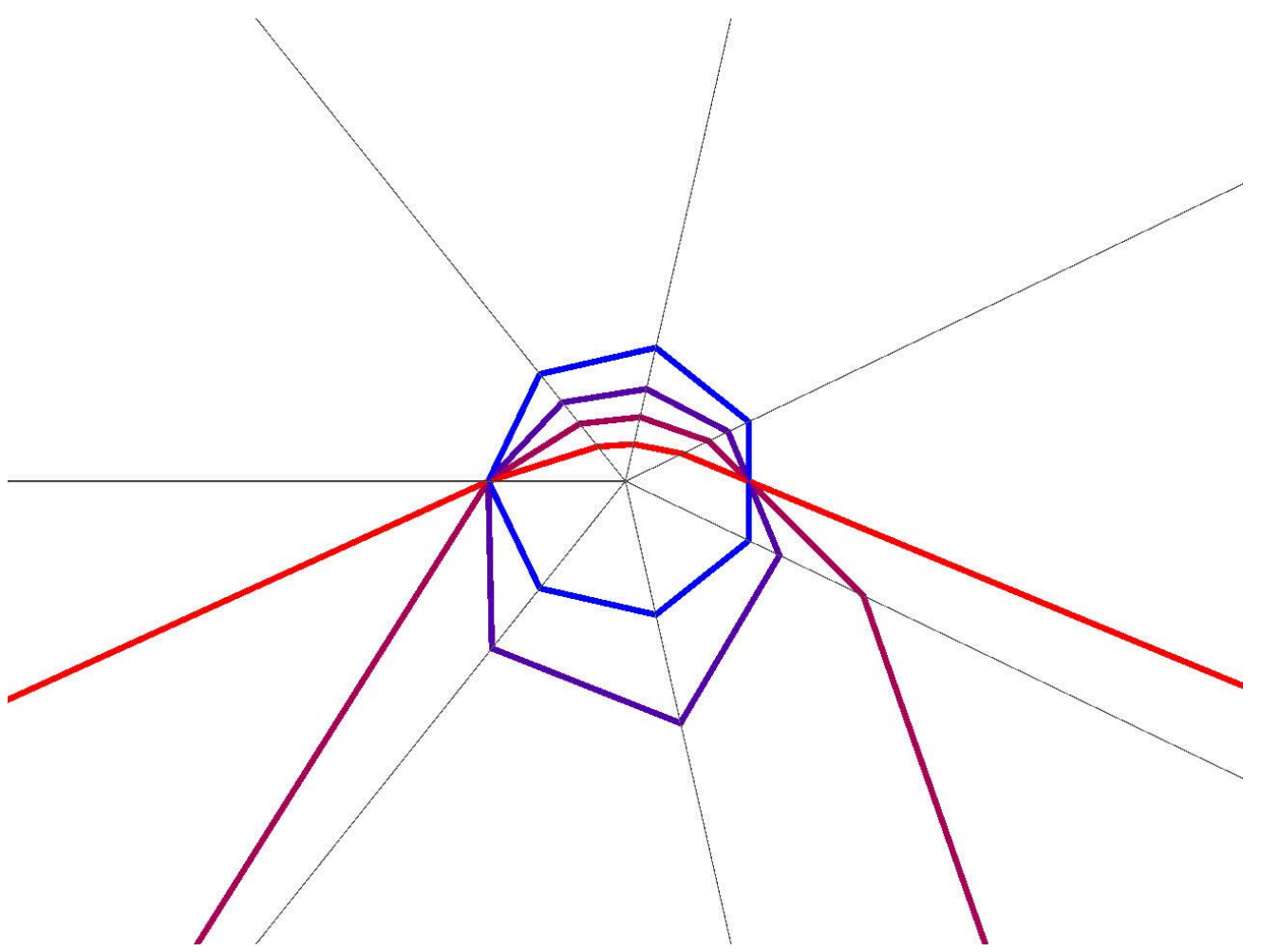

Figure 6. Trajectories inside a lens star with $N=7$ lenses. The image was created with Dr TIM. ${ }^{12}$

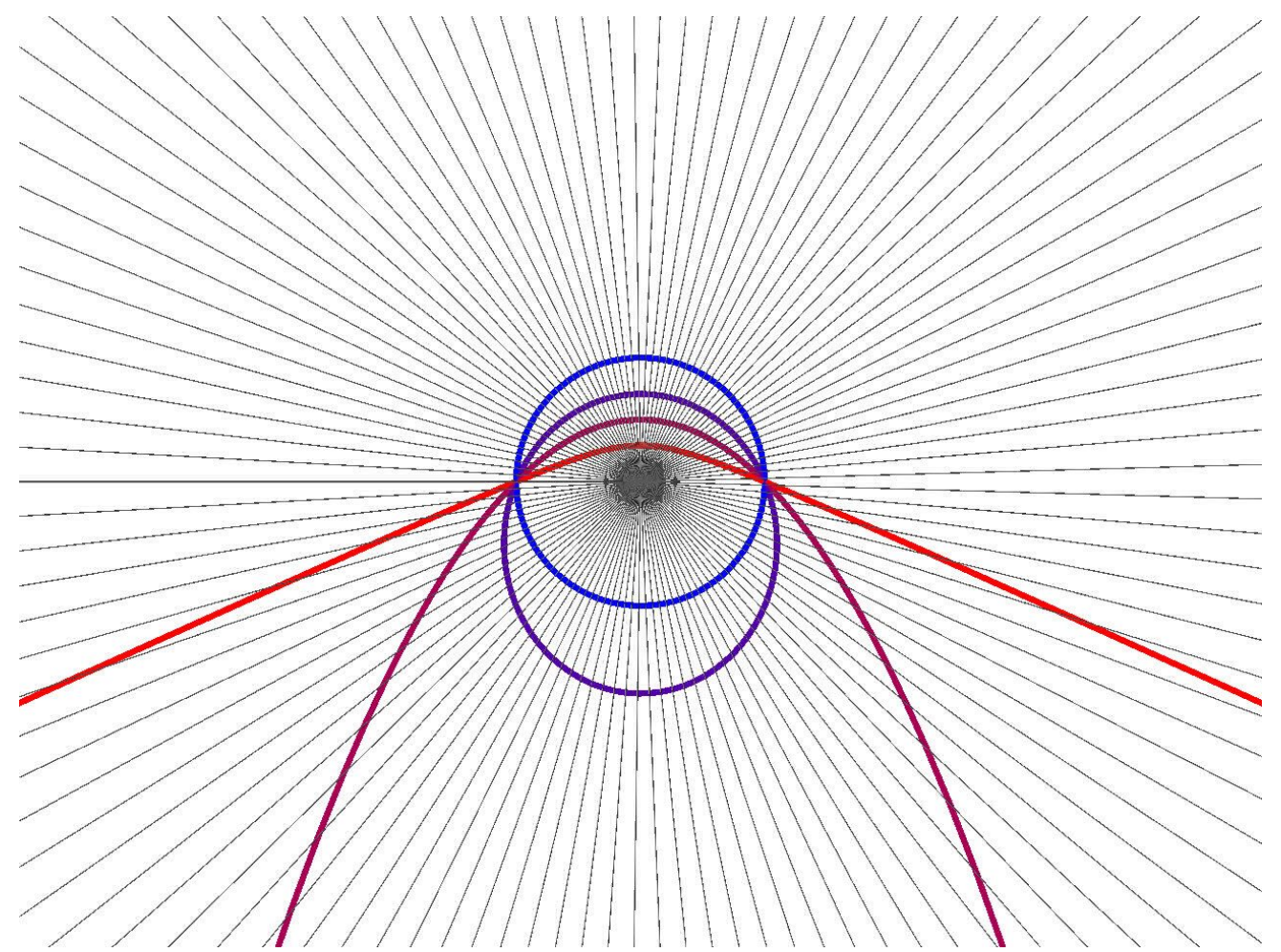

Figure 7. Trajectories inside a lens star with $N=113$ lenses. The image was created with Dr TIM. ${ }^{12}$ 


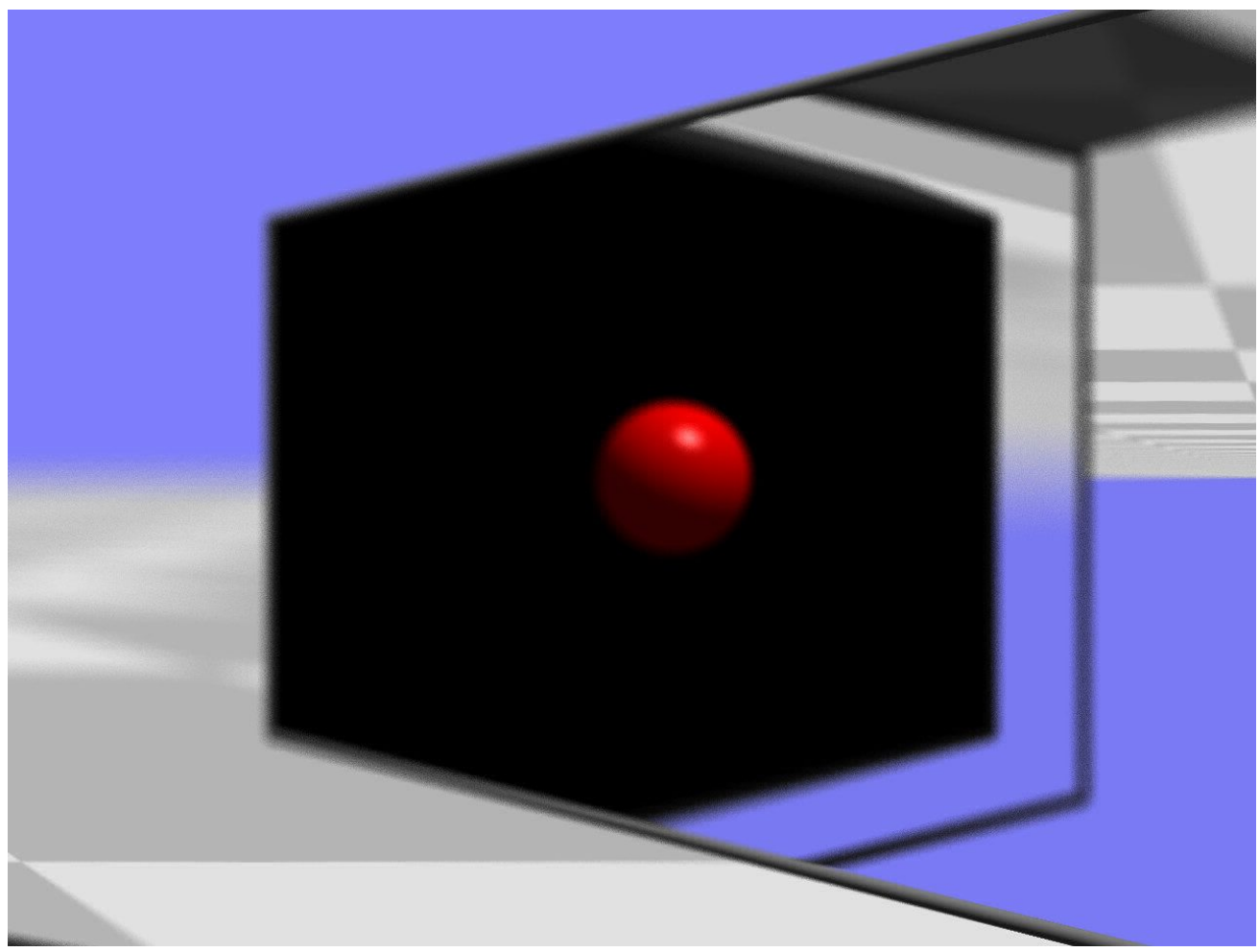

Figure 8. View from within the ideal-lens star shown in Fig. 4. The camera is centred on a point on the blue trajectory shown in The image was created with Dr TIM. ${ }^{12}$

[4] A. C. Hamilton and J. Courtial, "Generalized refraction using lenslet arrays," J. Opt. A: Pure Appl. Opt. 11, p. 065502, 2009.

[5] J. Courtial, "Standard and non-standard metarefraction with confocal lenslet arrays," Opt. Commun. 282, pp. 2634-2641, 2009.

[6] T. Maceina, G. Juzeliūnas, and J. Courtial, "Quantifying metarefraction with confocal lenslet arrays," Opt. Commun. 284, pp. 5008-5019, 2011.

[7] A. C. Hamilton and J. Courtial, "Metamaterials for light rays: ray optics without wave-optical analog in the ray-optics limit," New J. Phys. 11, p. 013042, 2009.

[8] J. Courtial and T. Tyc, "Generalised laws of refraction that can lead to wave-optically forbidden light-ray fields," J. Opt. Soc. Am. A 29, pp. 1407-1411, 2012.

[9] J. Courtial, "Geometric limits to geometric optical imaging with infinite, planar, non-absorbing sheets," Opt. Commun. 282, pp. 2480-2483, 2009.

[10] S. Oxburgh and J. Courtial, "Perfect imaging with planar interfaces," J. Opt. Soc. Am. A 30, pp. 2334-2338, 2013.

[11] S. Oxburgh, C. D. White, G. Antoniou, E. Orife, and J. Courtial, "Transformation optics with windows," Proc. SPIE 9193, p. 91931E, 2014.

[12] S. Oxburgh, T. Tyc, and J. Courtial, "Dr TIM: Ray-tracer TIM, with additional specialist capabilities," Comp. Phys. Commun. 185, pp. 1027-1037, 2014.

[13] T. Tyc, S. Oxburgh, E. N. Cowie, G. J. Chaplain, G. Macauley, C. D. White, and J. Courtial, "Omnidirectional transformation-optics cloak made from lenses and glenses," J. Opt. Soc. Am. A 33, pp. 10321040, 2016.

[14] S. Oxburgh, C. D. White, G. Antoniou, E. Orife, T. Sharpe, and J. Courtial, "Large-scale, white-light, transformation optics using integral imaging," J. Opt. 18, p. 044009, 2016. 
[15] G. J. Chaplain, G. Macauley, J. Bělín, T. Tyc, E. N. Cowie, and J. Courtial, "Ray optics of generalized lenses," J. Opt. Soc. Am. A 33, pp. 962-969, 2016.

[16] J. S. Choi and J. C. Howell, "Paraxial ray optics cloaking," Opt. Express 22, pp. 29465-29478, 2014. 